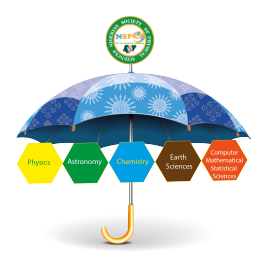

\title{
Review of Top Notch Electrode Arrays for Geoelectrical Resistivity Surveys
}

\author{
H. E. Ohaegbuchu ${ }^{\mathrm{a}, *}$, F. C. Anyadiegwu ${ }^{\mathrm{a}}$, P. O. Odoh ${ }^{\mathrm{a}}$, F. C. Orji ${ }^{\mathrm{a}}$ \\ ${ }^{a}$ Department of Physics, College of Physical and Applied Sciences, Michael Okpara University of Agriculture, Umudike, Nigeria.
}

\begin{abstract}
The different arrangements of electrodes used in geoelectrical resistivity surveys and measurements are referred to as electrode arrays. In this review, we have revisited most of the widely used electrode arrays as well as the uncommon ones, which are nonetheless, useful in certain situations. This review has provided detailed information about eleven (11) of the top notch electrode arrays employable in our regular resistivity surveys, making it clear that in practice, the arrays that are most commonly used for 2-D imaging surveys are the Wenner, Dipole-Dipole, Wenner-Schlumberger, Pole-Pole and the Pole-Dipole arrays. They have their strengths and weaknesses. They are typically described by their signal-to-noise ratio. Their depth of investigation, ability for lateral location of the target and their mapping abilities of horizontal layers or steeply dipping structures among other factors determine which array to adopt.
\end{abstract}

Keywords: electrode arrays, wenner, schlumberger, dipole-dipole, pole-dipole, resistivity surveys

Article History :

Received: 16 September 2019

Received in revised form: 08 January 2020

Accepted for publication: 11 January 2020

Published: 19 January 2020

(C)2019 Journal of the Nigerian Society of Physical Sciences. All rights reserved. Communicated by: O. J. Abimbola

\section{Introduction}

The purpose of electrical surveys is to determine the subsurface resistivity distribution by making measurements on the ground surface. From these measurements, the true resistivity of the subsurface can be estimated. The ground resistivity is related to various geological parameters such as the mineral and fluid content, porosity and degree of water saturation in the rock. Electrical resistivity surveys have been used for many decades in hydrogeological, mining and geotechnical investigations. More recently, it has been used for environmental surveys.

The resistivity measurements are normally made by injecting current into the ground through two current electrodes (A or

\footnotetext{
${ }^{*}$ Corresponding author tel. no: +2348132954367

Email address: eo.henry@mouau.edu.ng (H. E. Ohaegbuchu )
}

$\mathrm{C} 1$ and $\mathrm{B}$ or $\mathrm{C} 2$ ), and measuring the resulting voltage difference at two potential electrodes ( $\mathrm{M}$ or P1 and $\mathrm{N}$ or P2). From the current (I) and voltage $(\mathrm{V})$ values, an apparent resistivity $\left(\rho_{a}\right)$ value is calculated. The different arrangements of these electrodes are referred to as electrode arrays. Electrode arrays were developed in order to make field measurements more efficient and data interpretation easier. In this review, we will explore eleven (11) state-of-the-art electrode arrays that are employed for resistivity surveys, including the benefits, considerations, and applications of each array method.

\section{Wenner Array}

\subsection{Background}

The Wenner array was invented in 1915 by American physicist Frank Wenner (1873-1954), who worked at the American Bureau of Standards. His development of a four-terminal bridge 
design consisting of two outer current injection electrodes and two inner potential electrodes equally spaced became known as the "Wenner array" $[1,2]$.

\subsection{Field procedure}

The Wenner electrode array is the simplest of arrays; in it, the four electrodes - A, M, N, and B- are placed in line and spaced equidistant from each other. The two outer electrodes, $\mathrm{A}$ and $\mathrm{B}$, are current electrodes, and the two inner electrodes, $\mathrm{M}$ and $\mathrm{N}$, are potential electrodes. With the Wenner array, the resistivity of subsurface layers is found by increasing the distance between the electrodes while maintaining the location of the center point of the array [3]. The apparent resistivity measured by this array is given by equation 1 .

$$
\rho_{a}=2 \pi a \frac{\Delta V}{I},
$$

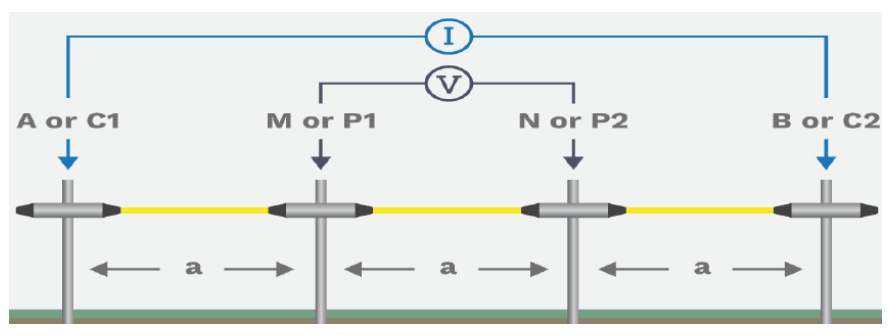

Figure 1: Wenner array (modified from Advanced Geoscience, Inc).

\subsection{Applications, Benefits, $\mathcal{E}$ Considerations Of The Wenner Array}

The Wenner array is commonly used in profiling for lateral exploration of the ground, like soil testing and sometimes VES for vertical exploration of the ground, like defining horizontal layers [1].

The Wenner array is cumbersome when performing VES surveys. Since you will have to move all four electrodes for each new measurement, this means a lot of walking when the electrode spacing becomes large. For example, to move all four electrodes from 50 meter electrode spacing to 100 meter electrode spacing, one person must walk a total of 600 meters for one measurement only-and a typical VES survey may comprise around 15 measurements. The alternative is to use more people; for example, one person at each electrode plus the instrument operator.

\section{Schlumberger Array}

\subsection{Background}

The Schlumberger array is named for Conrad Schlumberger, founder of the modern-day Schlumberger oilfield services company and pioneer of electrical methods in the early 1900s.

\subsection{Field procedure}

The Schlumberger array is an array where four electrodes are placed in line around a common midpoint. The two outer electrodes, A and B, are current electrodes, and the two inner electrodes, $\mathrm{M}$ and $\mathrm{N}$, are potential electrodes placed close together [4].

With the Schlumberger array, for each measurement the current electrodes $\mathrm{A}$ and $\mathrm{B}$ are moved outward to a greater separation throughout the survey, while the potential electrodes $\mathrm{M}$ and $\mathrm{N}$ stay in the same position until the observed voltage becomes too small to measure [5]. At this point, the potential electrodes $\mathrm{M}$ and $\mathrm{N}$ are moved outward to a new spacing. As a rule of the thumb, the reasonable distance between $\mathrm{M}$ and $\mathrm{N}$ should be equal or less than one-fifth of the distance between A and $\mathrm{B}$ at the beginning. This ratio goes about up to one-tenth or one-fifteenth depending on the signal strength. Equation 2 represents the apparent resistivity of the Schlumberger array.

$$
\rho_{a}=\frac{\frac{\pi\left(s^{2}-a^{2}\right)}{4}}{a} \frac{\Delta V}{I},
$$

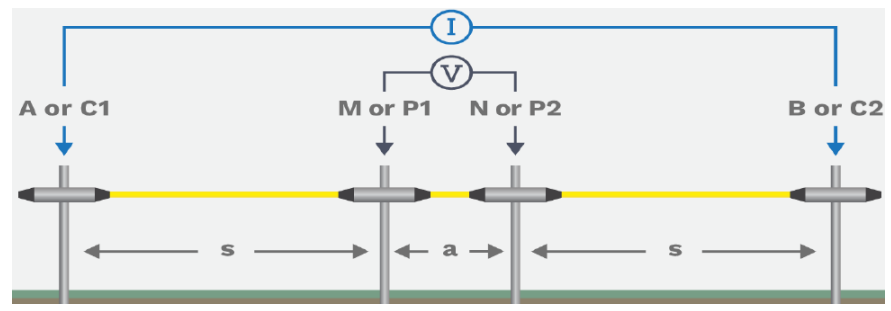

Figure 2: Schlumberger array (modified from Advanced Geoscience, Inc).

\subsection{Applications, Benefits, $\mathcal{E}$ Considerations Of The Schlum- berger Array}

The Schlumberger array is commonly used for vertical electrical sounding (VES) for groundwater and aggregate minerals. Vertical electrical sounding (VES) using the Schlumberger array provides better resolution, and take less time to deploy than the Wenner array [6].

The Schlumberger array is the best method for VES for practical reasons-it is significantly less labor-intensive than the Wenner array. For VES in general, the Schlumberger array is at advantage, because most of the time only the outer electrodes A and B need to be moved [4]. A crew of three people is normally enough for VES - with two people moving the outer electrodes, the instrument operator typically moves the inner electrodes the few times they need to be moved.

The Schlumberger array is also used for mapping or profiling for lateral resistivity changes. The typical profiling is done by larger fixed $\mathrm{AB}$ current electrode pairs and moving $\mathrm{MN}$ potential electrode pairs between them $[5,7,8]$. It is also done like Wenner profiling with fixed four ABMN electrode spacing The disadvantage of using profiling, regardless of using the Schlumberger or Wenner arrays, is that it demands homogeneous horizontal layers, which hardly ever occur naturally. 


\subsection{Potential Drawbacks}

- Substantial lengths of cable energized with current at high voltage present a safety hazard.

- The Schlumberger array is a labour-intensive survey because of the cable lengths required and the movement of the electrodes during the survey.

\section{Dipole-Dipole Array}

\subsection{Background}

The dipole-dipole array was originally used for mineral exploration with the induced polarization (IP) method. The dipoledipole method made it easier to visualize the collected data in the form of 2D handmade contour plots. Even though you couldn't get a perfect image of the ground, it gives you an approximate image which you could interpret the geology from [9].

As previously mentioned, the big change with the dipoledipole array came with automated resistivity and IP imaging (where raw data is collected automatically and inverted (computermodelled) into true IP and true resistivity, which we'll examine below.

\subsection{Field procedure}

A dipole is a pair of oppositely charged electrodes that are so close together that the electric field seems to form a single electric field rather than a field from two different electric poles. On the other hand, when the separation of the two charged electrodes is large enough that the observer detects the electric field from two poles, it's called a bipole [9].

The dipole-dipole array consists of a current electrode pair $\mathrm{A}$ and $\mathrm{B}$ and a potential electrode pair $\mathrm{M}$ and $\mathrm{N}$, and it offers a way to plot raw data in order to get an idea of a cross-section of the earth. Those using the dipole-dipole array look at a measurement value called apparent resistivity, which represents a weighted average of the resistivities under the four electrodes used to take the reading. The apparent resistivity is typically calculated by modern instruments from the geometry between the four electrodes and the injected current and measured potential [10].

The result of a dipole-dipole survey is plotted in a pseudosection. For each measurement, the apparent resistivity data is plotted at the midpoint between the two dipoles and at a depth half the distance between the two dipoles [10].

The value is finally contoured and colourized, which represents a rough and often severely distorted image of the subsurface. So while this array doesn't provide an actual image of the ground, the data points resulting from these measurements provide an image of the cross section that one can then try to interpret [11]. Today, modern inversion software can recalculate all of these apparent resistivity values to "true" resistivity values so that a realistic image of the ground can be created.

The primary advantages of the dipole-dipole array are its high resolution and multi-channel capability; it provides a very detailed image instead of providing a "big picture" image like the Wenner array.

The dipole-dipole array is fast because it supports multiple receiver channel measurements simultaneously (the AGI SuperSting R8 can measure eight simultaneous measurements for each current injection) whereas the Wenner array can only collect one data point for each current transmitter injection. Additionally, it is relatively simple to perform a survey along profile lines using the dipole-dipole array. The disadvantage of this array is that the dipoles will lose the signal if they're placed too far apart, thereby decreasing the ability to see deeper into the earth. The expression for the apparent resistivity is as follows:

$$
\rho_{a}=\pi n(n+1)(n+2) a \frac{\Delta V}{I}
$$

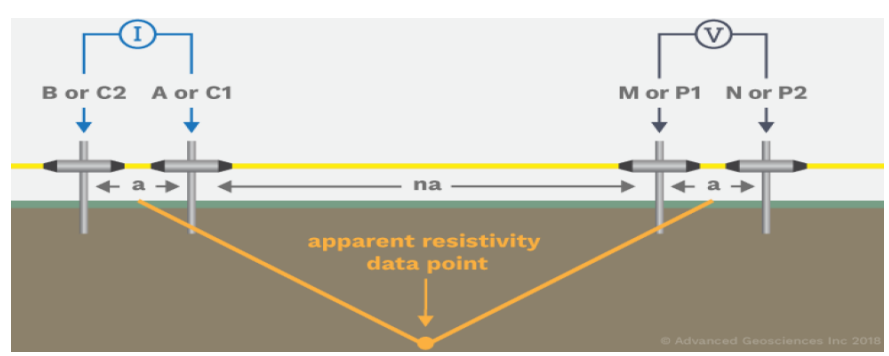

Figure 3: Dipole-Dipole array (modified from Advanced Geoscience, Inc).

\subsection{Using The Dipole-Dipole Array With Modern Resistivity $\mathcal{E}$ IP Scanning Instruments}

To conduct a survey using the dipole-dipole array, you first place a large number of electrode stakes out with equal spacing between the stakes. For example, you could have 100 electrodes, each spaced one meter apart. If this were the case, you'd have a 99 meter long profile. With spacings at 10 meters apart, you will have a 990 meter long profile. The longer the profile, the deeper you are able to see - at a cost of less detail in the image. After all the stainless steel electrode stakes are installed in the ground and connected with one multi-conductor cable to the receiver instrument, you then start the measuring sequence.

The electrodes in positions one and two are your current electrodes, labelled A and B. The electrodes in positions three and four are your potential electrodes, labelled $\mathrm{M}$ and $\mathrm{N}$. The instrument takes a reading referred to the mid-point between the current and potential dipoles. Once that is complete, the instrument automatically moves the potential electrodes ( $\mathrm{M}$ and $\mathrm{N})$ to the electrodes in positions four and five. (The electrodes stay in the same place physically, but the instrument connects with a different pair of electrodes).

This process repeats-electrodes A and B stay in positions one and two while $\mathrm{M}$ and $\mathrm{N}$ move out one electrode spacing for each reading. For every step out, the signal becomes weaker. At a certain point, the instrument can no longer take a reading. At this point, the instrument automatically moves the current electrodes A and B out a step-from positions one and two to positions two and three. This cycle continues throughout the 
electrode array. Using the SuperSting R8, you can conduct a field survey in roughly 15 minutes (instead of the two or more hours another system may take, which decreases the costs of time in the field).

Keep in mind that modern equipment and software allow you to perform multiple arrays at one time. Using the SuperSting instrument, you could perform a combination dipole-dipole and Schlumberger (gradient) array at once to allow you to see depth and detail even better.

\section{Pole-Dipole Array}

\subsection{Background}

The pole-dipole array has relatively good horizontal coverage, but it has a significantly higher signal strength compared with the dipole-dipole array and it is not as sensitive to telluric noise as the pole-pole array. Unlike the other common arrays, the pole- dipole array is an asymmetrical array and over symmetrical structures the apparent resistivity anomalies in the pseudo section are asymmetrical. In some situations, the asymmetry in the measured apparent resistivity values could influence the model obtained after inversion. One method to eliminate the effect of this asymmetry is to repeat the measurements with the electrodes arranged in the reverse manner. By combining the measurements with the "forward" and "reverse" poledipole arrays, any bias in the model due to the asymmetrical nature of this array would be removed.

\subsection{Field procedure}

A pole is a single transmitting electrode, and a dipole is a pair of oppositely charged electrodes that are so close together that the electric field seems to be a single electrode field instead of fields from two different electric poles.

The pole-dipole array is similar to the dipole-dipole array, but the pole-dipole array is used when the surveyor needs to see deep within a cross section of the earth [12]. The achievable depth is based entirely on the distance between the two electrodes (the dipole and the pole). The dipole-dipole array, on the other hand, is used to provide a very detailed image of a cross section of the earth-but will lose signal if the dipoles are placed too far apart. Like the dipole-dipole array, the poledipole array is most often used for mineral and ore exploration. The apparent resistivity is given as:

$$
\rho_{a}=2 \pi n(n+1) a \frac{\Delta V}{I},
$$

\subsection{Using The Pole-Dipole Array With Modern Resistivity $\mathcal{E}$ IP Scanning Instruments}

When conducting a survey using the pole-dipole array, the transmitting remote electrode is moved to infinity. In other words, the transmitter remote electrode should be so far away from the receiver dipole that the instrument does not sense the effect of the remote (pole) electrode, which is often 10 times the distance of the survey area. The reason for this is that the distance between the two electrodes determines the depth of

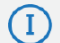

$\infty \mathrm{B}$ or $\infty \mathrm{C} 2$ 10x the distance of
the survey area

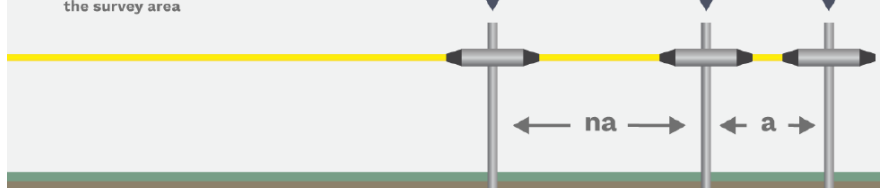

Figure 4: Pole-Dipole array (modified from Advanced Geoscience, Inc).

electrical penetration - and explains why the pole-dipole array is popular when you need to look deep into the earth.

This is a critical element because if the remote electrode is not far enough away from the dipole, the electrical current from the dipole to the pole will create a concentric circle of electricity (so long as the ground is homogeneous). If there is a geologic deviation in the ground, placing the remote electrode too close to the dipole will create a deviation in this concentric circle-but will still distort the measurements.

When correctly configured, you move the potential electrodes (the dipole) to a position near the current electrode (the pole), and take a reading. Then you move the dipole one step forward, and take another reading. You continue this until you begin losing signal between the current and the potential electrodes.

Keep in mind that modern equipment and software allows you to perform multiple arrays at one time. You could use the SuperSting instrument to perform a pole-dipole array.

\section{Pole-Pole Array}

\subsection{Field procedure}

The four electrodes in the pole-pole array are arranged so that the distance between the transmitter (A\&B) dipole and the receiver $(\mathrm{M} \& \mathrm{~N})$ dipole is small in comparison. In fact, the distance ought to be $10 \%$ that of the A\&B dipole [10].

In a pole-dipole survey, one transmitting electrode is moved away from the dipole to infinity (i.e., 10 times the distance of the survey area) so the instrument does not sense the pole electrode. In a pole-pole survey, one receiver electrode is also moved to infinity, but additionally, one of the potential electrodes is moved to infinity in the opposite direction. In other words, in a pole-pole array, you have a stationary infinity electrode on either side of the survey area.

Then proceed with your resistivity or induced polarization measurements exactly as you would with a pole-dipole array: Move the $\mathrm{A}$ and $\mathrm{M}$ electrodes to a new location inside the survey area and take a reading with a receiver instrument. The apparent resistivity according to [13] is:

$$
\rho_{a}=2 \pi n \frac{\Delta V}{I}
$$




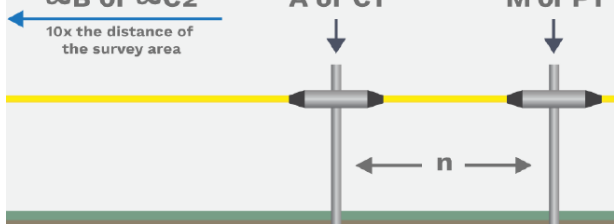

Figure 5: Pole-Pole array (modified from Advanced Geoscience, Inc).

\subsection{Considerations For Using The Pole-Pole Array}

The primary issue with using the pole-pole array is space-a problem that makes it far less common than both the dipoledipole array and the pole-dipole array. The two infinity electrodes are stationary, but you need to place them far out in opposite directions, and you may have to get permission to place them on someone else's land. You also have to consider hazards like traffic, creeks, or brush when handling the connecting wire. A large MN may pick up plenty of cultural, SP, and telluric noise.

For example, if you wanted to conduct a resistivity survey to look for groundwater at approximately 300-400 meters deep, you would need to have two kilometres between your electrodes. This is an enormous area to cover for a survey, and there are numerous things to consider in this circumstance, including the cost, the amount of time it takes to roll out the wire, and the labour necessary.

Furthermore, you have to be mindful of any physical or geographical hazards. For instance:

- You may need permission from the owner of neighbouring land to place your electrode over their boundary.

- You could be restricted if the electrode cable needs to cross a busy road.

- Bodies of water or vegetation may complicate matters.

\section{Equatorial Array}

\subsection{Field procedure}

Also known as the Equatorial-Dipole array, the Equatorial array consists of the two dipoles-A-B and $\mathrm{M}-\mathrm{N}$ - oriented perpendicular to the survey line.

During the sounding process, $\mathrm{M}-\mathrm{N}$ is moved away from A$\mathrm{B}$ along the perpendicular bisector (n). This perpendicular bisector may be regarded as an equator to the poles at A and B; hence the name "equatorial arrangement." [12].

\subsection{Applications, Benefits $\mathcal{E}$ Considerations}

The advantage of the Equatorial array is that you are able to penetrate even deeper into the ground than the Dipole-Dipole array (assuming the length along the survey line is the same). This can be particularly advantageous when pulling an array of electrodes behind a vehicle or in limited spaces [14].

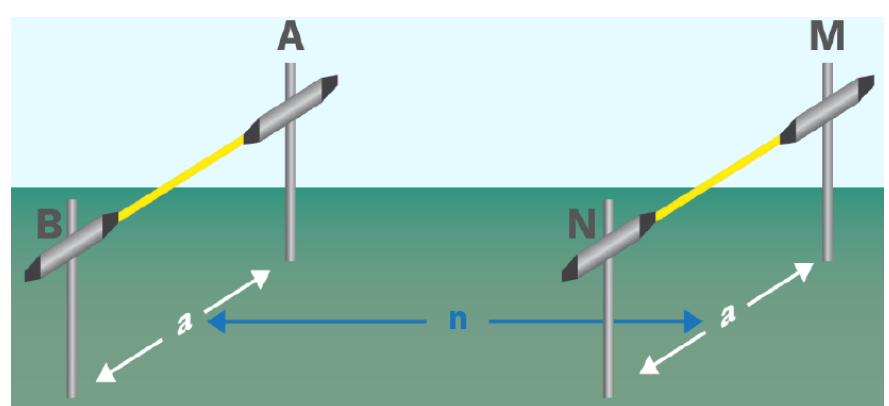

Figure 6: Equatorial array (modified from Advanced Geoscience, Inc).

One consideration of the Equatorial array is that even though it can see deeper than other arrays, it will lose signal as it is expanded, which limits its use.

The equatorial array is used less often than other arrays, but it is still very useful. It can be especially helpful in situations where you are limited by tight space constraints. For example, imagine doing a survey in a parking garage. Certainly, you do not have much space between support structures there. Using this array, you can see as deep as possible in a limited space.

\section{Square Array}

\subsection{Field procedure}

The Square array is a special case of the Equatorial array. The name derives from the electrodes forming the shape of a square-with the four sides of the array being of equal length. This array is used most frequently in determining anisotropy, or the resistivity in different directions of a geological formation. Instead of expanding the electrodes, in Square array surveys, the square is typically turned 15 degrees around its centre point for each measurement [15].

The apparent resistivity for the Square array depends on what ABMN geometry $(\mathrm{K})$ you use. Below is the generic formula for apparent resistivity to get you started.

$$
\rho_{a}=K \frac{\Delta V}{I},
$$

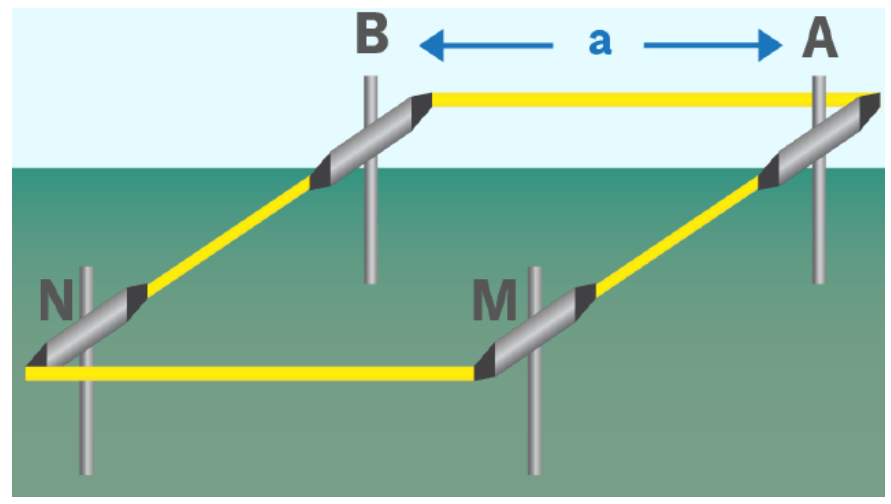

Figure 7: Square array (modified from Advanced Geoscience, Inc). 


\section{Examples of the Square Array}

(top-down view)
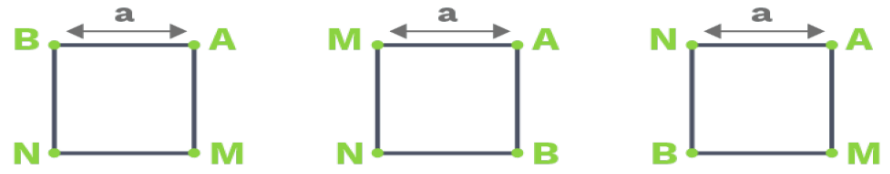

Figure 8: Square array (modified from Advanced Geoscience, Inc).

\subsection{Applications, Benefits $\mathcal{E}$ Consideration}

As with the Equatorial array, an advantage of the Square array is that you are able to penetrate deeper into the ground than when using the Dipole-Dipole array (assuming the length along the survey line is the same). As mentioned above, it is a good array for determining anisotropy.

With the development of 3D arrays, the need for the Square array has diminished over the years. It is often not used much in the U.S. any more, though it still has some popularity in other countries. With a 3D array, you could easily see clearer data than what is provided by the Square array. That said, the Square array is the cheaper option of the two. The Square array is usually only used if the field conditions are right and if the survey operator has some base knowledge of fractures.

Square array resistivity surveys would be a good fit for identifying environmental problems. Specifically, preferential flow paths and the way spills will flow and follow fractures [15].

Let us say you are a hydrogeologist that needs to survey a rock body with a fracture system. You will want to locate the general fracture direction. Taking a number of readings through the square array, you can determine which direction is more conductive and thus the main direction of the fractures.

Furthermore, if you needed to see deeper to gain more information on fractures in the rock body, you could expand the size of the square and repeat the same procedure. It may, for instance, show that the fracture changes direction at a particular depth.

\section{Gradient Array}

\subsection{Field procedure}

The Gradient array is similar to the Schlumberger array. The difference is that the Schlumberger array records only the center receiver dipole, whereas the gradient array measures all adjacent dipoles from one transmitter electrode to the other, including the center-most dipole [16].

To take a measurement with the Gradient array, a reading is taken of the potential field between two current electrodes side-by-side (which form a dipole). The next reading goes one step to the right and continues down the row of electrodes. This process repeats until you have mapped the whole area you are examining. The lateral changes in the potential field of the Gradient array are measured between the transmitter dipole $\mathrm{A} \& \mathrm{~B}$. Recording the lateral changes is often referred to as a profiling method [17].

\subsubsection{What is the difference between Edge Gradient and Strong Gradient?}

As mentioned above, the two forms of Gradient array that can be used for 2D profiles are the Edge Gradient and the Strong Gradient.

All measurements on an Edge Gradient use the "a" electrode spacing between the transmitters A\&B. Current electrodes-startin with the B-current electrode-remain at the first electrode until the A-current electrode reaches the last electrode on the 2D line. Then, the A-current electrode remains at the last electrode until the B-current electrode reaches the A-current electrode. This Gradient array generates a large number of measurements.

Alternatively, the measurements on a Strong Gradient is comprised of multiples of eight measurements. These are always taken between two transmitting electrodes with different "a" electrode spacing like a, 2a, 3a, 4a, etc.

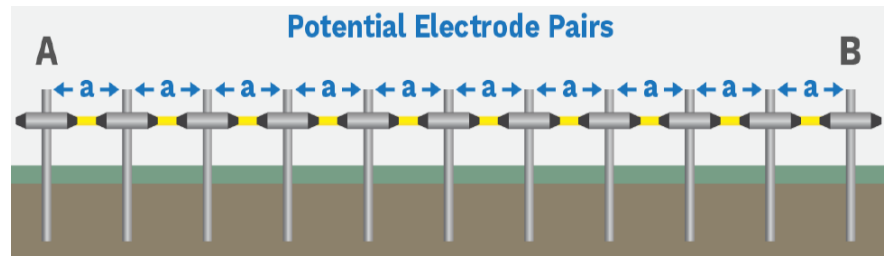

Figure 9: Gradient array (modified from Advanced Geoscience, Inc).

\subsection{Applications, Benefits $\mathcal{E}$ Considerations}

Profiling methods such as the Gradient array are ideal for locating sinkholes, fractures, and other geological changes. The Strong Gradient array is also great for conductive environments such as on the seafloor to enhance measured signal [18].

A full spread of 56 electrodes with one meter spacing between will give you a very strong signal near the two current electrodes using the Edge Gradient. (but a very weak signal in the center of your survey area). The Strong Gradient array is a good choice to improve such weak signal issues. Strong Gradient produces a strong signal and it is recommended for a large number of electrodes to reduce the number of measurements. This is one of the reasons we recommend the Strong Gradient for most geophysical surveys - even just as a backup array [17].

Historically, these arrays were considered to be impractical, slow, and hard to visualize. Before automatic multi-electrode systems, resistivity imaging instruments most often consisted of four electrodes connected to a voltmeter. Using the Gradient array with this type of system was very time consuming, as it requires someone to take readings and then move the electrodes until the whole survey area was mapped. But with modern multi-electrode resistivity imaging systems, you can automatically take measurements between each dipole for a quick and efficient survey [19].

Since Gradient arrays, especially the Edge Gradient array, frequently depend on the first and last electrodes on the survey line, it is highly recommended to closely observe the contact resistances on these electrodes during the entirety of your survey to reduce noisy data. 


\section{Azimuthal Method}

\subsection{Field procedure}

You may have noticed that we said "method" and not "array" at the beginning of this section. That is because the Azimuthal Method is less of an array and more of an electrode orientation or method-similar to vertical electrical sounding (VES), profiling, or Mise-a-la-Masse.

"Azimuth"-in relation to a geophysical survey-is the direction or angle of a particular location compared to north. You can measure azimuth with many different electrode arrays - the Wenner array being one of them.

With the Wenner array-an axial array-you set your current electrodes along the 12 O'clock and 6 O'clock and your inner electrodes on that line at a 10 meter electrode spacing. You then move your electrodes clockwise either 10 degrees or a quarter turn (depending on your preference) and take another reading. As you repeat this, your outer electrodes form a circle with a 30 meter diameter, and your inner electrodes form another circle with a 10 meter diameter. You can increase the Wenner electrode spacing to measure deeper [20].

Evaluation of this data gives you information about fracture patterns. The Wenner "a spacing" is associated with resistivity at different depth levels in the formation below, which is interpreted through what is called a polar diagram or rose diagrams. This diagram uses concentric circles (which represent resistivity value) and a north, south, east, and west axis. If anisotropy is present in the ground, then the polar diagram forms an ellipsoid figure when a line is drawn from each data point, as seen in the image below [2].

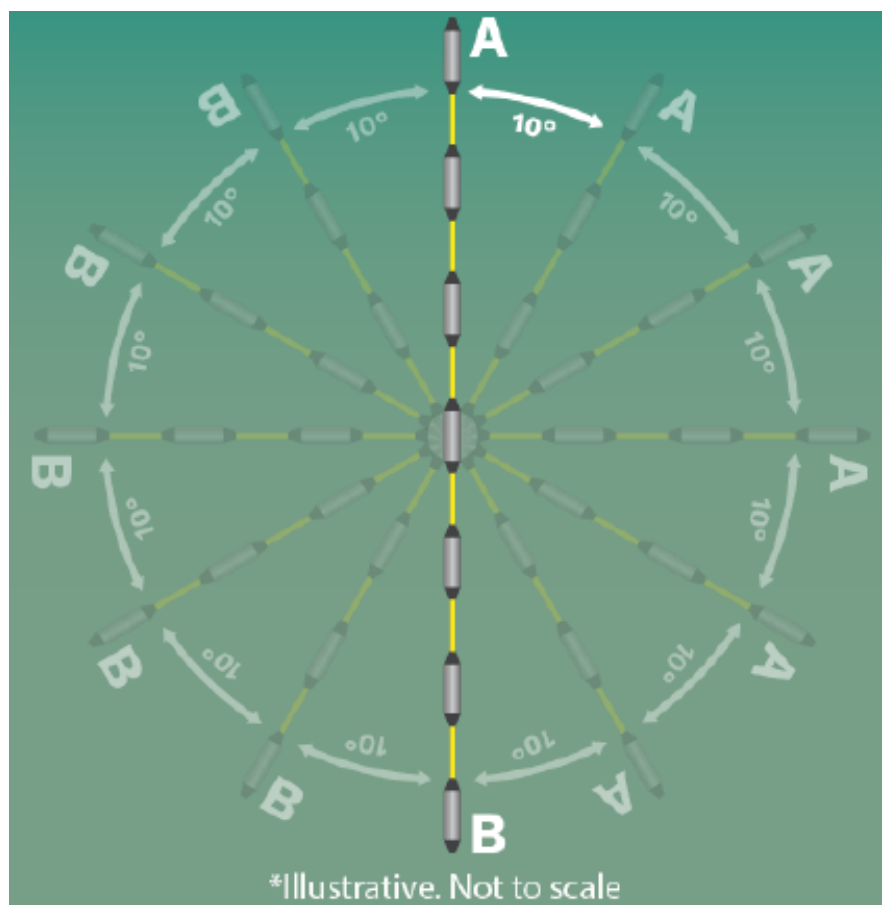

Figure 10: Azimuthal Method (modified from Advanced Geoscience, Inc).

\subsection{Applications, Benefits $\mathcal{E}$ Considerations}

Hydrogeologists can learn a great deal by applying this method to study fracture patterns and how water flows through them. Rock formations in the earth are anisotropic-meaning they have different resistivities based on the direction of the resistivity measurement. The changes in directional resistivity are often due to the fracture patterns in the subsurface [21].

So, for example, if a hazardous liquid is spilled, it may be critical to know the direction of the fractures in a particular area to contain the site-and electrical resistivity testing allows for a noninvasive way to "see" these fractures [22].

Consider an electrode array rotating around a center point. If there are fractures in the subsurface, at some point the electrode array will be parallel to the fractures. This happens because the fractures hold sediment, soil, clay, and moisture, which creates a more conductive path then the surrounding material. These fractures are termed heterogeneous features. Anisotropy is another directional component found in some soils which can have an azimuthal response as well [17]. Differentiating these two features requires core samples in many cases.

The Azimuthal Method may exhibit a response which looks like fractures, but in fact be due to a layer called a dipping bed. This is a flat layer of lower resistivity material which angles down away from the surface. This is because Azimuthal Method is a 1D VES or sounding method and thus cannot image the true shape of the bed or fracture like a 3D image would. 3D imaging is the most reliable way to measure fractures. Multiple parallel 2D lines can also be merged into a good 3D model for this same goal [23, 24].

As previously noted, the Wenner array is not the only array used with the Azimuthal Method-and it can actually be prone to issues. There are some who suggest that the Square array is more appropriate for azimuthal measurements, as it is the most sensitive for direction [25].

\section{Mise-A-La-Masse}

\subsection{Field procedure}

Like Vertical Electrical Sounding (VES) and profiling, or the Azimuthal Method-Mise-a-la-Masse is more of a method than an electrode array. You may have seen this method also called the "charged body potential method" or the "excitationat-the-mass method". Loosely translated from French, Mise-ala-Masse means "charged body" [26].

This method was very popular in the 1920's and 1930's for searching out ore bodies. In fact, it is still used today for the same reason-among others. If you are looking to use this method, the Pole-Dipole array is most frequently paired with Mise-a-la-Masse.

To perform this method, attach a current electrode (A) to an exposed portion of your conductive target-such as ore, water, or plumes. Because the target is conductive, it acts as a giant electrode. The current electrode (B) is placed at infinity-and the potential electrode $(\mathrm{N})$ is placed at infinity opposite of B. The $\mathrm{M}$ electrode is then used to detect the edges of the underground target $[27,28]$. 


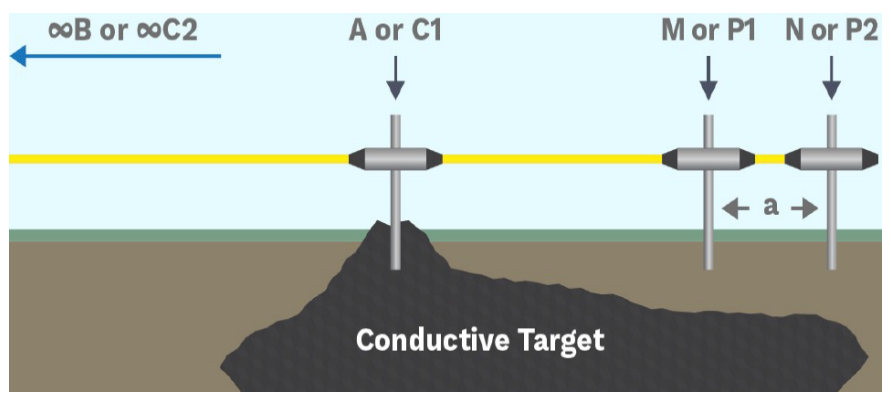

Figure 11: Mise-A-La-Masse array (modified from Advanced Geoscience, Inc).

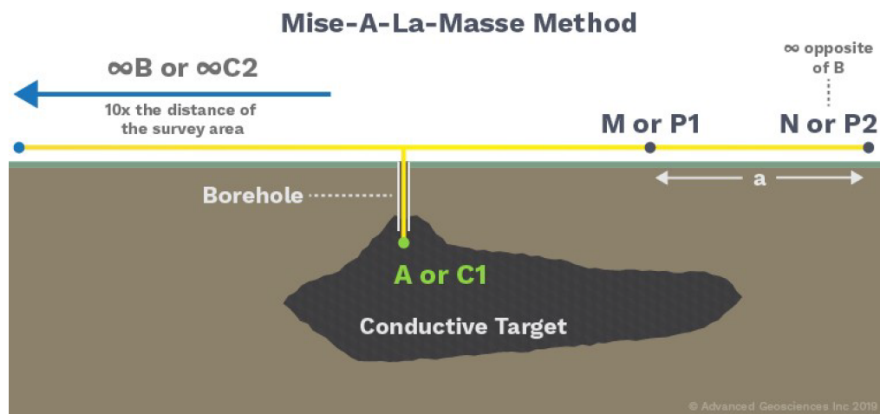

Figure 12: Mise-A-La-Masse array (modified from Advanced Geoscience, Inc).

\subsection{Applications, Benefits $\mathcal{E}$ Considerations}

Ore Body Exploration. The Mise-a-la-Masse method can be useful if an ore body is discovered during drilling. While you may know there is an ore body in the vicinity, you likely do not know its direction or how it extends underground. Using Misea-la-Masse can help you to be more precise in your exploration and excavation. For ore exploration, you would essentially follow the steps we outlined above- - though your current electrode (A) would be attached to a wire and lowered into a borehole to attach to an exposed portion of the ore body [28].

Detecting Pond Membrane Leakage. Mise-a-la-Masse can also be used to detect leakage in rubber pond membranes or liners. For this application, it is the same steps as stated above, except you will place your current electrode (A) near the edge of the pond in the water.

If there are no holes or leaks in the membrane, the instrument will not be able to send out the requested current when the current is injected into the water. But if a hole or leak is present-even if it is the size of a pinhole-the current from the electrode will find the hole and move toward the infinity electrode (B). This creates a source of electricity in the pond which can easily be located [27].

Mise-a-la-Masse was popular in the 20's and 30's for a reason. It is a relatively easy method to use, and it is a great option for the applications we have listed below. Though it is great for a specific use, it is not a method that you will need to use often.

\section{Bipole-Bipole Array}

\subsection{Field procedure}

It is easy to get this array confused with the Dipole-Dipole since they are similar. In fact, the field from the transmitting electrodes when using the Dipole-Dipole array is actually the field from a Bipole (especially as it pertains to the measurements closest to the transmitting electrodes).

The word "Bipole" is used instead of "Dipole" when the two transmitting electrodes are placed so far apart that the electric field from them can be considered a field from two separate poles. In other words, the distance between the receiver and transmitter dipoles is small in relation to the dipoles themselves [29].

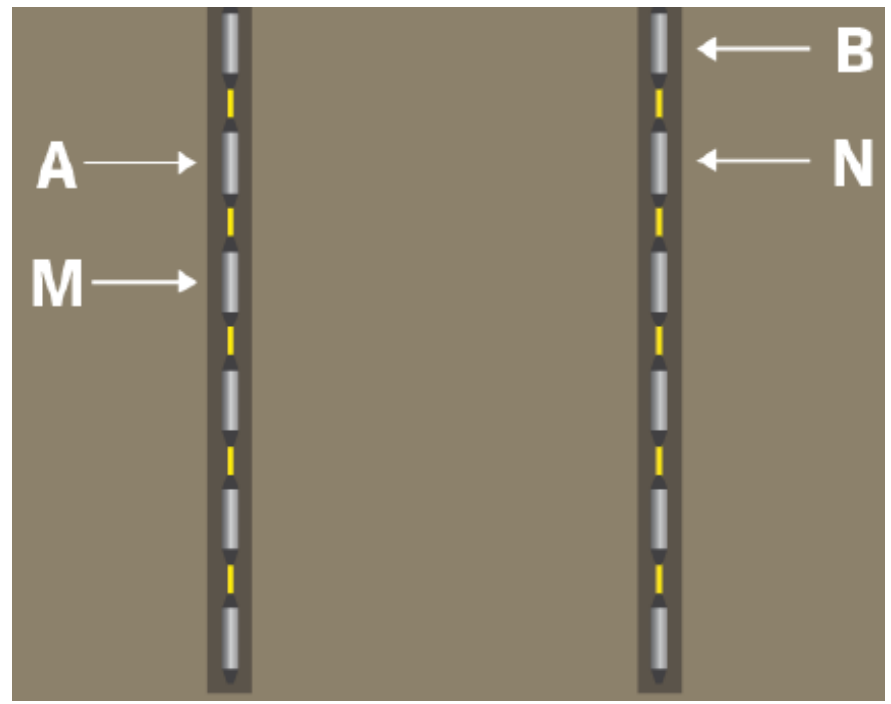

Figure 13: Bipole-Bipole array (modified from Advanced Geoscience, Inc).

\subsection{Applications, Benefits $\mathcal{E}$ Considerations}

As mentioned above, the Bipole-Bipole array is useful for some uncommon 3D geometries. For instance, if you want to install electrodes surrounding a large building, or structure. You would want to use Bipole-Bipole instead of Dipole-Dipole because the current and voltage electrodes are going to be far apart.

The Bipole-Bipole array becomes very useful on cross-borehole studies for strong current injection, where generally, DipoleDipole fails to do so. It is also useful for some uncommon 3D geometries.

Though it is also possible to use the Bipole-Bipole array for any mix of surface and depth electrodes (electrodes installed within the earth materials) - you cannot use this array unless some of the electrodes in your geometry have a negative Zvalue. So if using this array, be prepared to install electrodes beneath the surface via boreholes or some other method [29]. 


\section{Conclusion}

Having reviewed the various electrodes designs for geoelectrical resistivity surveys, we conclude by noting that in practice, the arrays that are most commonly used for 2-D imaging surveys are the (a) Wenner, (b) dipole-dipole (c) WennerSchlumberger (d) pole-pole and (e) poledipole. These arrays are commonly used in resistivity surveys. They have their strengths and their weaknesses. They are typically described by their signal-to-noise ratio. Their depth of investigation, their ability for lateral location of the target and their mapping abilities of horizontal layers or steeply dipping structures among other factors determine which array to adopt.

\section{Acknowledgments}

We thank the referees for the positive enlightening comments and suggestions, which have greatly helped us in making improvements to this paper.

\section{References}

[1] T. Dahlin, The development of a cable system for vertical electrical sounding and a comparison of the Schlumberger and offset Wenner methods, Licentiate thesis. Lund University, (1989) pp77.

[2] F. H. Al-Menshed, Evaluation of resistivity method in delineation ground water hydrocarbon contamination southwest of Karbala city, $\mathrm{PhD}$ Thesis, Dept. of Geology, College of Science, University of Baghdad (2011) pp210.

[3] G. V. Keller \& F. C. Frischknecht, Electrical Methods in Geophysical Prospecting, Pergamon Press, New York, (1966).

[4] J. M. Al-Ani, Theoretical and application considering the effect of the geometry on Schlumberger and dipole configurations, $\mathrm{PhD}$ thesis (in Arabic)", University of Baghdad, College of Science, (1998) pp144.

[5] W. M. Telford, L. P. Geldart \& R. E. Sheriff, Applied geophysics, Cambridge University Press, Cambridge, England, (1990) pp770.

[6] D. W. Oldenburg, "A quantitative technique for modeling ionospheric and magnetospheric current distributions", 83 (1978) 3320.

[7] K. Deppermann, "Die Abhangigkeit des scheinbaren Widerstandes vom Sondenabstand bei der Vierpunkt-Methode", Geophys. Prosp. 2 (1954) 262.

[8] A. A. Zohdy, "A new method for the automatic interpretation of Schlumberger and Wenner sounding curves", Geophysics 54 (1989) 245.

[9] A. Roy, "Depth of investigation in Wenner, three electrode and dipole dipole DC resistivity methods" Geophysical Prospecting 20329.
[10] L. M. Alpin, The Theory of Dipole Soundings, Gostoptekhizdat, Moscow (1950).

[11] G. Kunetz, Principle of direct current resistivity prospecting, Borntrager, Berlin (1966) pp106.

[12] A. A. Zohdy, Geometric factors of Bipole-Dipole arrays, U.S. Geological Survey, New Techniques in Direct-Current Resistivity Exploration (1970).

[13] C. A. Heiland, Geophysical exploration, Prentice-Hall, New York (1940) pp1013.

[14] R. Barker, "The offset system of electrical resistivity sounding and its use with a multi-core cable", Geophysical Prospecting 29 (1989) 128.

[15] G. M. Habbberjam, Apparent resistivity observations and the use of square array techniques Geoexploration Monographs 1 (1979) pp152.

[16] T. Dahlin \& C. Bernstone, "A roll-along technique for 3D resistivity data acquisition with multi-electrode array" Proceedings of the Symposium on the Application of geophysics to Engineering and Environmental Problems, Reno, Nevada 2 (1997) 927.

[17] B. Steinich, I. Simon, J. A. Chavarria, \& L. E. Marin, "Geological investigations of the vadose zone in the valley of Hermosillo aquifer, Sonora, Mexico" Geofisica Internacional, 36 (1997) 191.

[18] A. A. Zohdy, G. P. Eaton, \& D. R. Mabey, Application of surface geophysics to ground-water investigations, U.S. Geological Survey, Techniques of water-resource investigations, (1974) pp116.

[19] R. G. Van Nostrand \& K. L. Cook, (1966). "Interpretation of resistivity data" USGS Professional Paper, U.S. Govt 499 (1966).

[20] F. H. AL-Menshed, "How To Understand Theoretical Background Of Electrical Resistivity Method In A Simple Way" (2018) doi: 10.1007/11020-1102-5_6.

[21] P. Sjodahl, Resistivity investigation and monitoring for detection of internal erosion and anomalous seepage in embankment dams, Doctoral Thesis, Lund University, (2006) pp86.

[22] J. P. Busby, "The effectiveness of azimuthal apparent-resistivity measurements as a method for determining fracture strike orientations" Geophysical Prospecting 48 (2000) 677.

[23] R. W. Taylor \& A. H. Fleming, "Characterizing jointed systems by azimuthal resistivity surveys" Ground Water 26 (1988) 464.

[24] T. Dahlin \& B. Zhou, "A numerical comparison of 2D resistivity imaging with 10 electrode arrays" Geophysical Prospecting 52 (2004) 379.

[25] K. R. Nunn, R. D. Barker \& D. Bamford, "In-situ seismic and electrical measurements of fracture anisotropy in the Lincolnshire chalk" Quart. J. Eng. Geol. 16 (1983) 187.

[26] J. L. Osiensky, "Ground water modeling of mise-a-la-masse delineation of contaminated ground water plumes" J. Hydrol. 197 (1997) 146.

[27] P. K. Battacharya \& H. P. Petra, Direct current geoelectrical sounding, Elsevier Publishing Co., Amsterdam, (1968) p131.

[28] A. Binley \& A. Kemna, "DC Resistivity and Induced Polarization Methods" (2005) doi: 10.1007/1-4020-3102-5_5.

[29] S. Szalai, A. Koppan \& L. Szarka, "Effect of positional inaccuracies on multielectrode results" 13th European Meeting of Environmental and Engineering Geophysics, Istanbul, turkey (2007). 